\title{
Perceptions of Modulatory Factors in Migraine and Epilepsy: A Multicenter Study
}

\author{
Emel Ur Özçelik ${ }^{1 * \dagger}$, Katia Lin ${ }^{2 \dagger}$, Ruta Mameniškienè ${ }^{3}$, Juiane Sauter Dalbem ${ }^{2,4}$, \\ Heloise Helena Siqueira ${ }^{2,4}$, Rūta Samaitienè ${ }^{5}$, Luz Eleonora Vega Zeissig ${ }^{6}$, \\ Armando Ferreira Fonseca $\mathrm{Jr}^{2}$, Juliana Mazini Alves ${ }^{2}$, Mariana dos Santos Lunardi ${ }^{2}$, \\ Luiz Paulo de Queiroz ${ }^{2}$, Erika Zubavičiūtè ${ }^{7}$, Peter Wolf ${ }^{2,8}$ and Betül Baykan ${ }^{1}$

\begin{abstract}
${ }^{1}$ Departments of Neurology and Clinical Neurophysiology, Istanbul Faculty of Medicine, Istanbul University, Istanbul, Turkey, ${ }^{2}$ Department of Neurology, Universidade Federal de Santa Catarina, Florianópolis, Brazil, ${ }^{3}$ Centre for Neurology, Vilnius University, Vilnius, Lithuania, ${ }^{4}$ Department of Neurology, Universidade Federal de Mato Grosso, Cuiabá, Brazil, ${ }^{5}$ Faculty of Medicine, Clinic of Children's Diseases, Vilnius University, Vilnius, Lithuania, ${ }^{6}$ Centro de Epilepsia Humana y Neurocirurgia Funcional, Guatemala City, Guatemala, ${ }^{7}$ Faculty of Medicine, Vilnius University, Vilnius, Lithuania, ${ }^{8}$ Danish Epilepsy Center, Dianalund, Denmark
\end{abstract}

\section{OPEN ACCESS}

Edited by:

Rashid Giniatullin,

University of Eastern Finland, Finland

Reviewed by:

Paola Torelli,

University of Parma, Italy

Aleksey V. Zaitsev,

Institute of Evolutionary Physiology

and Biochemistry (RAS), Russia

*Correspondence:

Emel Ur Özçelik

emeluscas@gmail.com

†These authors have contributed equally and share first authorship

Specialty section:

This article was submitted to

Epilepsy,

a section of the journal

Frontiers in Neurology

Received: 26 February 2021

Accepted: 12 April 2021

Published: 03 June 2021

Citation:

Ur Özçelik E, Lin K, Mameniškienè R,

Sauter Dalbem J, Siqueira HH,

Samaitienè $R$, Vega Zeissig LE,

Fonseca AF Jr, Mazini Alves J, dos

Santos Lunardi $M$, de Queiroz LP

Zubavičiūtè $E$, Wolf $P$ and Baykan $B$

(2021) Perceptions of Modulatory

Factors in Migraine and Epilepsy: A

Multicenter Study.

Front. Neurol. 12:672860.

doi: 10.3389/fneur.2021.672860
Background: Migraine and epilepsy are both common episodic disorders, typically precipitated or inhibited by some modulatory factors (MFs).

Objective: To assess the self-perception of MFs in patients with migraine (PWM) compared to patients with epilepsy (PWE) with a standardized protocol in different countries.

Methods: Transcultural multicenter comparative cross-sectional study. All consecutive patients who fulfilled the ICHD-3 criteria for migraine and ILAE's criteria for epilepsy, with at least 1 year of follow-up were interviewed with a semi-structured questionnaire on clinical and epidemiological data and were asked to identify all experienced MFs from a provided list.

Results: A total of 608 individuals were surveyed at five university referral centers in Brazil, Guatemala, Lithuania and Turkey. Two hundred and nineteen (91.6\%) PWM and 305 (82.7\%) PWE identified attack precipitating factors (PFs; $p<0.001$ ). The most frequent three PFs reported by epilepsy patients were: "lack of sleep" (56.6\%), "emotional stress" (55.3\%), "negative feelings" (53.9\%), while among migraine patients "emotional stress" (81.6\%), "lack of sleep" (77.8\%), "negative feelings" (75.7\%) were cited. Inhibitory factors (IFs) for the episodes were reported by 68 (28.5\%) PWM and 116 (31.4\%) PWE. "Darkness" was the most common one, described by 35.6\% of PWM whereas "positive feelings" reported by $10.6 \%$ of PWE. Most MFs are concordant across the countries but some transcultural differences were noted.

Conclusion: The MFs of migraine and epilepsy attacks and their varying frequencies according to different countries were investigated with the same standardized questionnaire, for the first time. MFs were recognized very often in both migraine and epilepsy cohorts, but in distinct disease-specific prevalence, being more frequent in migraine. Recognition of self-perceived MFs may be helpful for the management of both illnesses.

\footnotetext{
Keywords: migraine, epilepsy, exogenous modulators, precipitant factors, inhibitory factors, self-awareness
} 


\section{INTRODUCTION}

Migraine and epilepsy are the two most common paroxysmal disorders of the central nervous system, with a prevalence of $\sim 12 \%$ or more $(1,2)$, and $\sim 1-2 \%(3,4)$, respectively. Different as they are, they have traits in common. One of them is the challenge to cope with the unpredictability of episodes. To know factors that may facilitate or provoke an attack, especially avoidable factors, would be a great help, and many patients attempt to identify them as well as factors having the opposite effect of preventing or inhibiting their paroxysmal events (5). Their views on such modulatory factors (MFs) have been the object of several investigations. In these, the two conditions were studied separately with different questionnaires, preventing a direct comparison, which would be interesting for various reasons. First, clear differences between the two heterogeneous conditions would be expected. Then, both could have certain traits in common like influences of stress or menstruation. Finally, there could be both unexpected differences and likenesses that could provide new insights into the experience of living with a paroxysmal disorder and suggest new hypotheses on the pathophysiology of the respective attacks.

We decided to conduct for the first time a comparative investigation of both disorders with the same instrument. As a second objective of this study, we compared the MFs between subgroups of epilepsy (focal vs. generalized) and migraine (with aura vs. without aura). The transcultural design allowed us, in addition, to identify possible national or regional traditions. Our working hypothesis was that it would be possible to identify different, condition-specific patterns and views of modulation of paroxysms. The focus of our study is on patients' experiences rather than the identification of objective such modulators, although their existence seems today well-established by prospective investigations of both conditions $(6,7)$.

\section{METHODS}

\section{Subjects and Questionnaire}

A multicenter observational study was undertaken in four university referral centers for neurological disorders located in four different countries, where total of 608 patients was interviewed from February 2016 to December 2019. All consecutive patients, who met the existing valid diagnostic criteria at the beginning of the study, were re-evaluated according to the new diagnostic criteria. The patients who fulfilled either the International Classification of Headache Disorders 3rd EditionICHD-3 (2018) criteria for episodic migraine or International League Against Epilepsy-ILAE's (2017) criteria for focal or generalized epilepsy were included for further analyses $(8,9)$. All participants should have at least 1 year of follow-up to secure the correct diagnoses and experienced a minimum of two attacks.

Abbreviations: MFs, modulatory factors; IFs, inhibitory factors; PFs, precipitating factors; PWM, patients with migraine; PWE, patients with epilepsy; MWA, migraine with aura; MWOA, migraine without aura.
For a comparative study in migraine and epilepsy, the same instrument needed to be used for both. As this was not available, we developed a new questionnaire in several expert discussion rounds and based on the existing questionnaires (Supplementary Table 1). It was physician-administered in a semi-structured face-to-face interview. Patients were asked to identify from this list all factors (MFs) that they perceived to be associated with the occurrence of seizures/migraine episodes.

Given that, in this neglected field, there is at present no generally accepted terminology, we defined "modulation" as including both "precipitating factors (PFs)" and "inhibitory factors (IFs)." Triggers pointed out to the specific and rare sensory or cognitive factors immediately precipitating reflex epileptic seizures, which are not specifically sought for this study.

Patients were excluded from this protocol if they were $<15$ years old, had any cognitive deficit that could prevent them from understanding the questionnaire, had evidence of progressive structural central nervous system lesions or progressive encephalopathy, had coexisting conditions (epilepsy and migraine), and had non-epileptic events, such as psychogenic seizures.

For subgroup comparisons of migraine patients, those with at least two attacks with aura were included in the migraine with aura (MWA) group, and the others were included in the migraine without aura (MWOA) group. Epilepsy patients were divided into two main groups as focal and generalized epilepsy according to their syndromic diagnoses by experts, based on their electroclinical features.

This study was carried out in accordance with the Code of Ethics of the World Medical Association (Declaration of Helsinki, 2014); and institutional review boards and ethics committees for each site approved the study protocol: Universidade Federal de Santa Catarina Ethics Committee, CEPSH/UFSC N. 1.226.636 (14/09/2015); Istanbul Medical Faculty Ethics Committee, no. 26/02/16/262-04; Investigation Committee of the Epilepsy and Functional Neurosurgery Center Humana CENFHU-06-2015; and Vilnius Regional Biomedical Research Ethics Committee no. 158200-15-797-309, 2015-09-07. All subjects signed an informed consent form and voluntarily agreed to participate.

\section{Statistical Analyses}

Statistical analysis was performed using IBM SPSS ${ }^{\circledR}$ Statistics Grad Pack software Premium version 26.0. Descriptive statistics were used to describe study population characteristics. Quantitative variables were expressed as mean \pm standard deviation (SD), and qualitative variables were expressed as frequency and percentage values. The Shapiro-Wilk normality test was used to test the normality of the distribution of quantitative data. The independent samples $t$-test was used to compare normally distributed continuous variables, whereas the Mann-Whitney $U$ and Kruskal-Wallis $H$-tests were used for variables not normally distributed. Pearson chi-square and Fisher's exact tests were used to compare categorical variables and frequencies of occurrence. While $p<0.05$ value was considered statistically significant for epilepsy and migraine comparisons, Bonferroni-corrected $p$-value $(0.05 / 2 ; p<0.025)$ 
was considered significant for epilepsy and migraine subgroup comparisons. When the results between the comparisons were statistically significant, the parameter in the chi-square boxes that created the significance was determined according to the adjusted values; a value of $\geq 2$ was considered as significant. In addition, the significant results for the most discordant MFs between various countries evaluated for triggers cited by more than 15 individuals to avoid bias.

\section{RESULTS}

Among the total of 608 subjects, 369 (60.7\%) had epilepsy and 239 (39.3\%) had migraine. The mean age was $33.24 \pm$ 12.92 years (range 15-83), and 396 individuals (65.1\%) were women (Table 1). The age distribution, duration of disease, and frequency of episodes were comparable, whereas the epilepsy group had an earlier age at onset and a lower rate of females. The main diagnoses were as follows: MWOA (53.6\%), MWA (43.8\%), and chronic migraine $(2.6 \%)$ among patients with migraine (PWM); and focal epilepsy (64.5\%) and generalized epilepsy (35.5\%) among patients with epilepsy (PWE).

The occurrence of PFs appeared very common in both groups, but it was reported more often with migraine (91.6\%) than with epilepsy (82.7\%, $p<0.001$, Figure 1A). Likewise, patients with both conditions experienced IFs in comparable but much lower frequency. IFs were not reported by 171 (71.5\%) PWM and 253 (68.6\%) PWE (Figure 1B). Additionally, the time lapse between the stimulus and the seizure or migraine episode is detailed in Table 2.

Moreover, substantial numbers of 82 (22.8\%) PWE and $45(18.8 \%)$ PWM declared to be able to prevent or arrest their episodes (Figure 2), whereas the counterpart, i.e., the ability to provoke attacks, was reported by
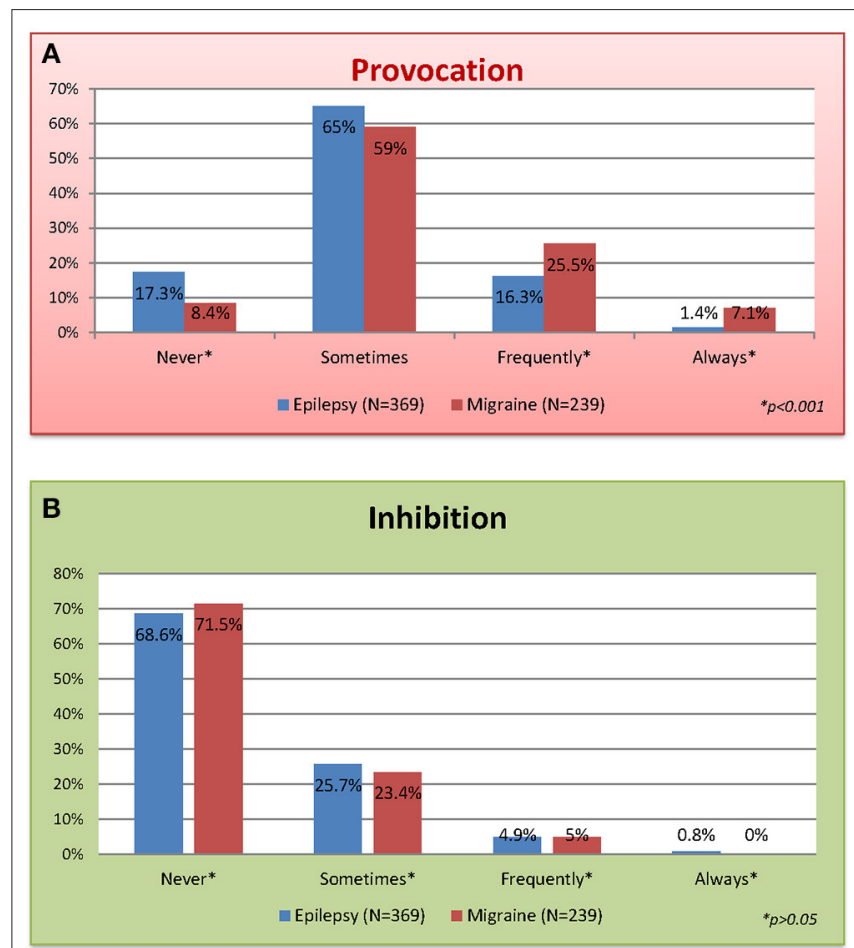

FIGURE 1 | Lifetime self-reported modulation of seizures or migraine episodes: (A) provocation and (B) inhibition.

TABLE 1 | Clinical and demographic information.

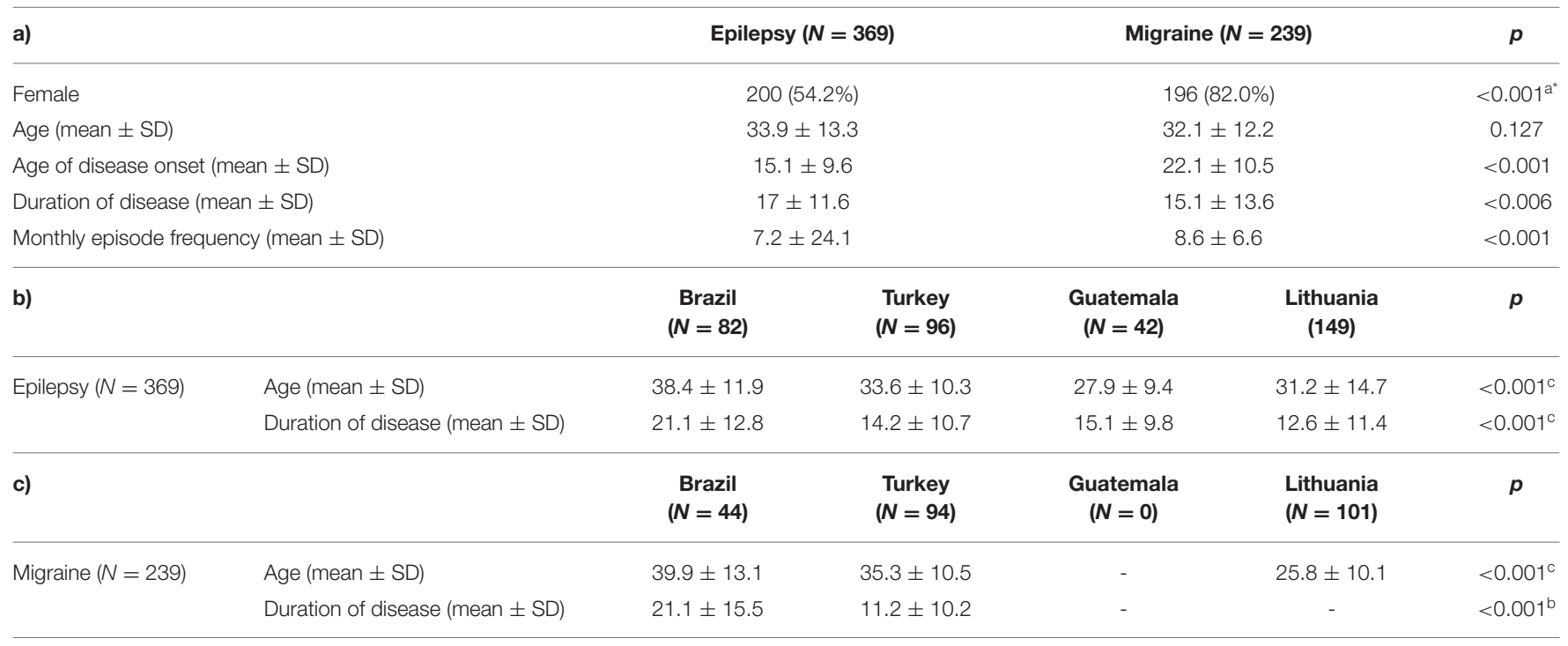

$S D$, standard deviation.

aPearson's chi-square.

${ }^{\text {b} M a n n-W h i t n e y ~ U-t e s t . ~}$

${ }^{c}$ Kruskal-Wallis H-test.

*Statistically significant $(p<0.05)$. 
$16.3 \%$ of PWM and, significantly less frequently, 5\% of PWE.

There were statistically significant differences between the epilepsy and migraine groups regarding the various types of PFs $(p<0.05)$. Migraineurs were more sensitive to many of those triggers related to emotional and physical conditions, concentration-required activities, and also sleep, hormonal, and dietary changes. The comprehensive table detailing all PFs and IFs from the checklist can be found as Supplementary Table 2, and we will report here the leading items.

The five main PFs reported were as follows: lack of sleep (56.6\%), emotional stress (55.3\%), negative feelings (53.9\%), physical stress (29.5\%), and certain thoughts (20.3\%) by epilepsy patients, while emotional stress $(81.6 \%)$, lack of sleep $(77.8 \%)$, negative feelings $(75.7 \%)$, hunger $(71.1 \%)$, and fasting (59\%) by migraine patients. Several other PFs appeared rather common in both conditions but with different weight. Provocation of attacks with alcohol reported by $42.1 \%$ of PWM and $21.5 \%$ of PWE, flickering lights by $52.7 \%$ of PWM and $18.7 \%$ of PWE, television

TABLE 2 | Delay between the stimulus and the episode of seizure/migraine.

\begin{tabular}{lcc}
\hline Time & $\begin{array}{c}\text { Epilepsy } \\
(\mathbf{N}=\mathbf{3 6 9 )} \%\end{array}$ & $\begin{array}{c}\text { Migraine } \\
(\mathbf{N}=\mathbf{2 3 9}) \%\end{array}$ \\
\hline Unknown* & $\underline{\mathbf{1 8 . 4}}$ & 11.7 \\
Immediate & 8.7 & 8.4 \\
Seconds & 9.8 & 10.5 \\
Minutes & 22.8 & 20.1 \\
Hours* & 13.8 & $\mathbf{3 3 . 9}$ \\
Variable & 19 & 15.1 \\
Not applicable & 7.5 & 0.3
\end{tabular}

The bold and underlined ones show statistically significant difference between the columns according to the adjusted residual $(\geq 2)$ values in Pearson's chi-square. ${ }^{*} p<0.001$. by $29.7 \%$ of PWM and $11.2 \%$ of PWE, and computer work by $43.5 \%$ of PWM and $13 \%$ of PWE. Excess sleep was reported by $54.8 \%$ of PWM but only $8.4 \%$ of PWE and fever by $30.1 \%$ of PWM and $13.5 \%$ of PWE. Menstruation was reported more frequently among migraineurs with active menstruation $(68.4 \%$ of PWM vs. $18.9 \%$ of PWE).

The rate of patients reporting at least two PFs was $81.6 \%$ in PWE, while it was $96.3 \%$ in PWM. Among migraineurs, the rate of those who reported at least 10 or more PFs was $72 \%$, while in PWE, this rate was only $20.1 \%$. There was a statistically significant difference between the number of reported factors per patient between two groups $(p<0.001)$ (Figure 3).

The main IFs reported by epilepsy patients were as follows: positive feelings (10.6\%), thinking/concentration (7.3\%), drawing (4.1\%), sports (4.1\%), and mental calculation (3.8\%). The rates of IFs among migraine patients were as follows: darkness (35.6\%), closing the eyes (31.4\%), bathing/shower/hot water $(18.4 \%)$, positive feelings $(16.3 \%)$, and coffee $(16.4 \%)$. The number of IFs reported per patient ranged from 1 to 17 in both groups, while the proportion of those reporting at least two IFs was $4.1 \%$ in PWE and $12.6 \%$ in PWM. The number of IFs reported per patient in migraine patients was significantly higher than in PWE $(p<0.001)$.

There was no significant difference between male and female genders, in terms of reporting provocation by triggers for their episodes in patients with both epilepsy and migraine $(p=0.298$, $p=0.105$, respectively). Also, there was no significant difference between women and men with epilepsy who reported that their attacks were inhibited by various factors $(p=0.561)$. However, the rate of men who reported that their migraine attacks were never inhibited by any factor (88.4\%) was significantly higher than women $(67.9 \%)(p=0.005)$.

To compare the effect of age on triggers, PWE were divided into two groups: under 32 years old and above, according to the median value of the whole group. There was no statistically significant difference between the two age groups in terms of

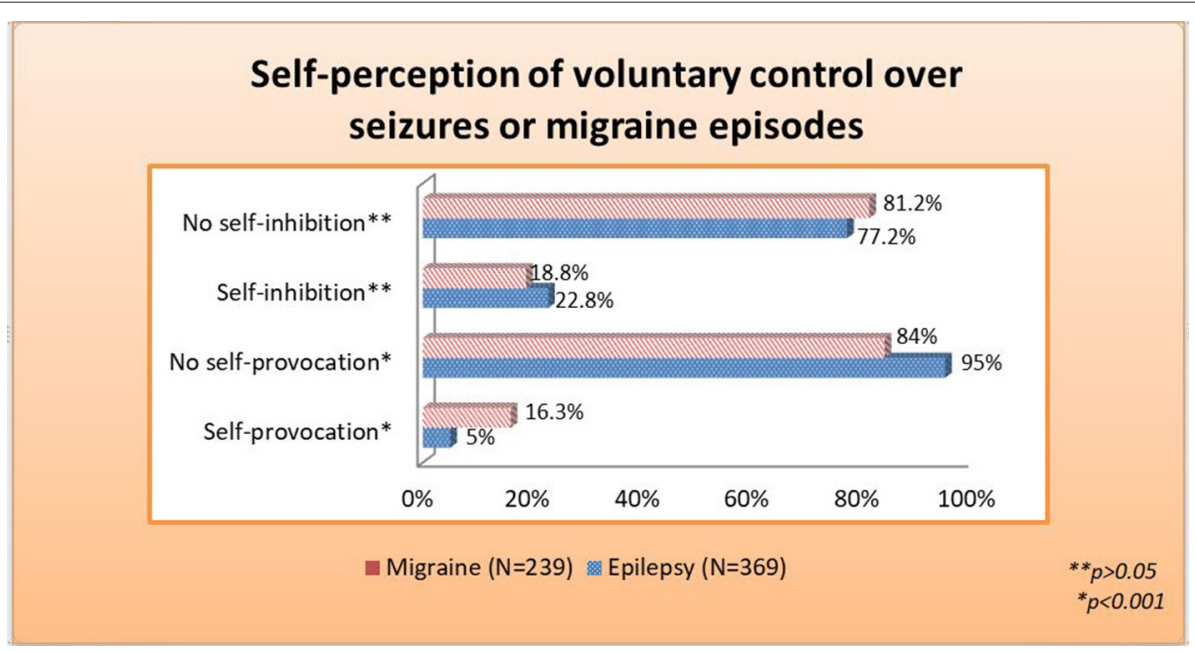

FIGURE 2 | Self-perception of voluntary control over seizures or migraine episodes. 


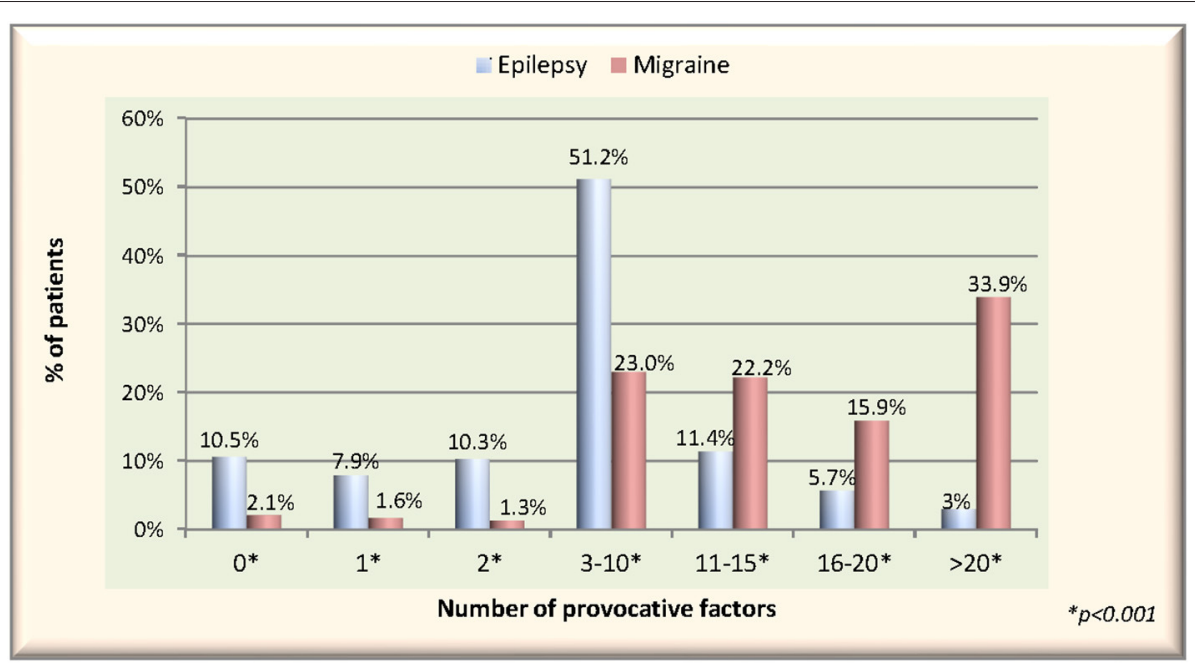

FIGURE 3 | The rates of reported number of provocative factors per patient in migraine vs. epilepsy groups.

TABLE 3 | Modulatory factors with significant difference between two age groups in migraine.

\begin{tabular}{|c|c|c|c|c|c|c|}
\hline & \multicolumn{2}{|c|}{$\begin{array}{c}\text { PWM } \leq 30 \text { years } \\
(N=122)\end{array}$} & \multirow[b]{2}{*}{ NoMod\% } & \multicolumn{2}{|c|}{$\begin{array}{c}\text { PWM > } 30 \text { years } \\
(N=117)\end{array}$} & \multirow[b]{2}{*}{ NoMod\% } \\
\hline & Provoc\% & Inhibit\% & & Provoc\% & Inhibit\% & \\
\hline Positive feelings ${ }^{\star \star}$ & 3.3 & 23.0 & $\underline{73.8}$ & 8.5 & 9.4 & 82.1 \\
\hline Pleasant taste ${ }^{\star \star}$ & 8.2 & $\underline{9.8}$ & 82 & 12.8 & 0.9 & 86.3 \\
\hline Chewing $\underline{p}=0.000^{*}$ & $\underline{17.2}$ & 4.9 & 77.9 & 3.4 & 0.9 & 95.7 \\
\hline Unpleasant aroma** & 30.2 & - & $\underline{69.8}$ & $\underline{60}$ & - & 40 \\
\hline Specific voices ${ }^{\star \star}$ & $\underline{29.5}$ & - & 70.5 & 14.5 & - & 85.5 \\
\hline Certain rhythms ${ }^{\star \star}$ & 15.6 & $\underline{5.7}$ & 78.7 & 18.8 & 0 & 81.2 \\
\hline Pain* & $\underline{44.3}$ & 3.3 & 52.5 & 26.5 & 0 & $\underline{73.5}$ \\
\hline Public speaking* & 32.8 & 0 & 67.2 & 12.8 & 0.9 & $\underline{86.3}$ \\
\hline Writing* & $\underline{69.6}$ & $\underline{6.6}$ & 80.3 & 30.4 & 0 & $\underline{94}$ \\
\hline Drawing ${ }^{\star \star}$ & 3.3 & $\underline{9.8}$ & 49.1 & 6 & 0 & 50.9 \\
\hline Singing** & 8.2 & $\underline{7.4}$ & 84.4 & 5.1 & 0.9 & 90.6 \\
\hline Sexual activity** & 6.8 & $\underline{16.2}$ & 76.9 & 12.3 & 3.5 & 84.2 \\
\hline Orgasm** & 6 & $\underline{16.2}$ & 77.8 & 6 & 3.5 & 89.5 \\
\hline Excess sleep ${ }^{\star \star}$ & 63.9 & 0.8 & 35.2 & 45.3 & 0 & 54.7 \\
\hline Seizures while asleep ${ }^{\star \star}$ & 37.7 & 5.7 & 56.6 & 19.7 & 5.1 & 75.2 \\
\hline Fever* & 42.6 & 0.8 & 56.6 & 17.1 & 0 & 82.9 \\
\hline
\end{tabular}

A comparison of the two groups here was made according to the median age of migraineurs. Statistical calculations were done among those who were exposed to modulatory factors.

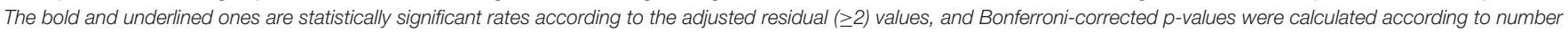
of migraine subgroups $(0.05 / 2 ; p<0.025)$ in Pearson chi-square.

Provoc, provocation; Inhibit, inhibition; NoMod, no modulation.

${ }^{\star} p \leq 0.001$.

${ }^{\star \star} p<0.025$.

reporting provocation and inhibition; $21.3 \%$ of those above the age of 32 years and $11.6 \%$ of below the age of 32 stated that their attacks were frequently provoked by various factors $(p=$ 0.083). Statistically significant ones are given in Table 3. Also, migraineurs were divided into two groups under the age of 30 and above, according to the median value of the whole group. Although there was no difference in terms of the responses given when asked about the rates of provoked and inhibited attacks (0.972), it was noteworthy that the young migraineurs were clearly more sensitive when all the MFs were questioned one by one. Statistically significant ones are given in Table 4 .

The top of the reported MFs according to country are given in Figure 4. While for PWE, emotional stress/negative feelings and lack of sleep were the most concordant PFs 
TABLE 4 | Modulatory factors with significant difference between two age groups in epilepsy.

\begin{tabular}{|c|c|c|c|c|c|c|}
\hline & \multicolumn{3}{|c|}{$\begin{array}{c}\text { PWE } \leq 32 \text { years } \\
(N=190)^{\neq}\end{array}$} & \multicolumn{3}{|c|}{$\begin{array}{c}\text { PWE }>32 \text { years } \\
\quad(N=178)^{\neq}\end{array}$} \\
\hline & Provoc\% & Inhibit\% & NoMod\% & Provoc\% & Inhibit\% & NoMod $\%$ \\
\hline Certain memories* & 12.1 & 2.6 & 85.3 & $\underline{26.4}$ & 0 & 73.6 \\
\hline Certain thoughts ${ }^{\star \star}$ & 14.2 & 3.2 & 82.6 & $\underline{26.4}$ & 1.1 & 72.5 \\
\hline Negative feelings ${ }^{\star \star}$ & 46.8 & 1.1 & $\underline{52.1}$ & $\underline{61.2}$ & 0 & 38.8 \\
\hline Lights $^{\star \star}$ & $\underline{23.7}$ & 0.5 & 75.8 & 13.5 & 0 & 86.5 \\
\hline $\mathrm{TV}^{*}$ & $\underline{15.3}$ & 1.1 & 83.7 & 6.2 & 0 & 93.8 \\
\hline Listening to talks, audit. overexposure ${ }^{\star \star}$ & 3.7 & 1.6 & 94.7 & $\underline{11.2}$ & 0 & 88.8 \\
\hline Videogames, playst., game boy ${ }^{\star \star}$ & $\underline{16.4}$ & 1.6 & 82 & 4.1 & 0 & 95 \\
\hline Working on computer** & $\underline{18.7}$ & 3.3 & 78 & 8.7 & 1.2 & 90 \\
\hline Dancing $^{\star \star}$ & 2.2 & $\underline{3.2}$ & 94.6 & 0.6 & 0 & 99.4 \\
\hline Sexual activity* & 1.7 & $\underline{2.8}$ & 95.5 & $\underline{7.4}$ & 0 & 92.6 \\
\hline
\end{tabular}

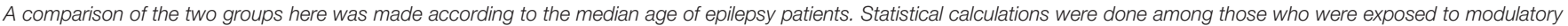

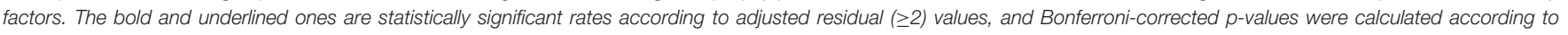
number of epilepsy subgroups $(0.05 / 2 ; p<0.025)$ in Pearson chi-square.

Provoc, provocation; Inhibit, inhibition; NoMod, no modulation; Audit, auditory; Playst, PlayStation.

${ }^{*} p \leq 0.001$.

${ }^{* \star} p<0.025$.

$\neq$ Missing age data for one patient.

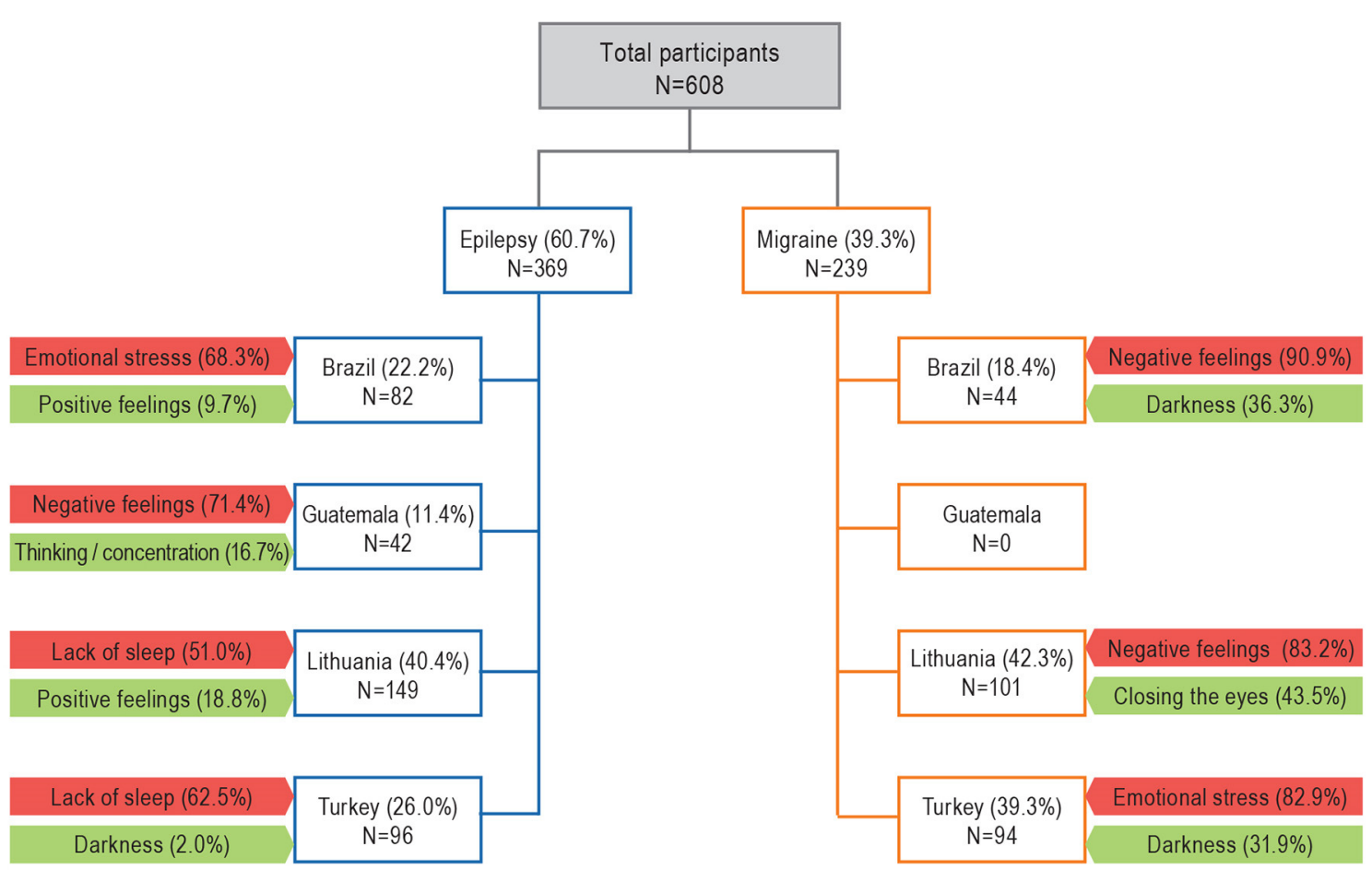

FIGURE 4 | Interviewed patients and most frequently reported modulatory factors according to country. The red-painted boxes show the rate of those reporting "provocations," whereas the green ones show the rate of reported "inhibitions".

among countries, positive feelings were the most concordant common IF. Similarly, for PWM, emotional stress/negative feelings were the most concordant common PFs among countries, whereas darkness was the most concordant common IF.
In subgroup comparisons of epilepsy and migraine, there were also statistically significant differences for the various types of MFs. Lack of sleep and lights were the leading triggers reported among generalized epilepsies, whereas emotional and mnemonic ones were frequent among 
TABLE 5 | Modulatory factors with significant difference between focal and generalized epilepsy.

\begin{tabular}{|c|c|c|c|c|c|c|}
\hline & \multicolumn{2}{|c|}{$\begin{array}{c}\text { Focal } \\
(N=238)\end{array}$} & \multirow[b]{2}{*}{ NoMod \% } & \multicolumn{2}{|c|}{$\begin{array}{l}\text { Generalized } \\
\qquad(N=131)\end{array}$} & \multirow[b]{2}{*}{ NoMod \% } \\
\hline & Provoc $\%$ & Inhibit \% & & Provoc $\%$ & Inhibit\% & \\
\hline Certain memories* & 24.4 & 0.8 & 74.8 & 9.9 & 2.3 & 87.8 \\
\hline Certain thoughts ${ }^{\star \star}$ & 25.2 & 1.3 & 73.5 & 11.5 & 3.8 & 84.7 \\
\hline Déjà vu* & 19.7 & 0 & 80.3 & 7.6 & 1.5 & 90.8 \\
\hline Positive feelings ${ }^{\star *}$ & $\underline{13}$ & 10.1 & 76.9 & 2.3 & 11.5 & 86.3 \\
\hline Lights* & 13 & 0.4 & $\underline{86.6}$ & $\underline{29}$ & 0 & 71 \\
\hline Flashes $^{*}$ & 12.2 & 0.8 & 87 & 30.5 & 0 & 69.5 \\
\hline Brightness ${ }^{\star \star}$ & 5.9 & 0.4 & 93.7 & 14.5 & 0 & 85.5 \\
\hline Listening to talks ${ }^{\star *}$ & $\underline{9.7}$ & 0 & 90.3 & 3.8 & $\underline{2.3}$ & 93.9 \\
\hline Sports ${ }^{\star \star}$ & $\underline{16}$ & 3.8 & 80.3 & 3.8 & 4.6 & $\underline{91.6}$ \\
\hline Lack of sleep ${ }^{\star \star}$ & 50.8 & 0.8 & 48.3 & 67.2 & 1.5 & 31.3 \\
\hline Seizure upon awakening* & 12.6 & 0 & 87.4 & $\underline{28.2}$ & 1.5 & 70.2 \\
\hline Substance use ${ }^{\star \star}$ & 4.5 & 0 & 95.5 & 3.9 & $\underline{5.3}$ & 90.8 \\
\hline
\end{tabular}

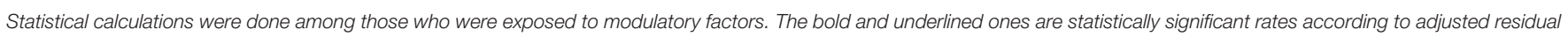
$(\geq 2)$ values, and Bonferroni-corrected $p$-values were calculated according to number of epilepsy subgroups $(0.05 / 2 ; p<0.025)$ in Pearson chi-square.

Provoc, provocation; Inhibit, inhibition; NoMod, no modulation.

${ }^{*} p \leq 0.001$.

${ }^{* *} p<0.025$.

TABLE 6 | Modulatory factors with significant difference between migraine with and without aura.

\begin{tabular}{|c|c|c|c|c|c|c|}
\hline & \multicolumn{3}{|c|}{$\begin{array}{l}\text { MWOA }^{\neq} \\
(N=133)\end{array}$} & \multicolumn{3}{|c|}{$\begin{array}{c}\text { MWA }^{\neq} \\
(N=104)\end{array}$} \\
\hline & Provoc $\%$ & Inhibit\% & NoMod\% & Provoc $\%$ & Inhibit \% & NoMod \% \\
\hline Certain memories ${ }^{\star}$ & 9 & 0 & $\underline{91}$ & $\underline{21.2}$ & 1 & 77.9 \\
\hline Chewing* & 6.8 & 0.8 & 92.5 & 15.4 & 5.8 & 78.8 \\
\hline Unpleasant taste* & 5.3 & 1.5 & $\underline{93.2}$ & $\underline{15.4}$ & 0 & 84.6 \\
\hline Lights ${ }^{\star \star}$ & 41.4 & 0 & $\underline{58.6}$ & 68.3 & 1 & 30.8 \\
\hline Flashes $^{\star \star}$ & 30.1 & 0 & $\underline{69.9}$ & $\underline{52.9}$ & 1 & 46.2 \\
\hline Brightness ${ }^{\star}$ & 30.1 & 0.8 & 69.2 & $\underline{48.1}$ & 1 & 51 \\
\hline Striped patterns* & 14.3 & 0.8 & $\underline{85}$ & $\underline{29.8}$ & 0 & 70.2 \\
\hline Any song ${ }^{\star \star}$ & 6.8 & 0 & 93.2 & 31.7 & 0 & 68.3 \\
\hline Certain rhythms ${ }^{*}$ & 12 & 1.5 & 86.5 & $\underline{24}$ & 4.8 & 71.2 \\
\hline Chess, cards, other* & 4.2 & 0 & $\underline{95.8}$ & 11.9 & $\underline{3.6}$ & 84.5 \\
\hline Sexual activity* & 6.9 & 6.2 & $\underline{86.9}$ & 13.1 & $\underline{15.2}$ & 71.7 \\
\hline Orgasm* & 4.6 & 6.2 & 89.2 & 9.1 & 15.2 & 75.8 \\
\hline Physical stress ${ }^{\star}$ & 50.4 & 0 & 49.6 & 64.4 & 1.9 & 33.7 \\
\hline
\end{tabular}

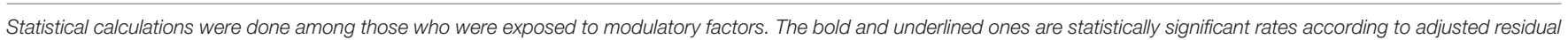
$(\geq 2)$ values, and Bonferroni-corrected $p$-values were calculated according to number of migraine subgroups $(0.05 / 2 ; p<0.025)$ in Pearson chi-square.

MWOA, migraine without aura; MWA, migraine with aura; Provoc, provocation; Inhibit, inhibition; NoMod, no modulation.

$\neq$ Two uncertain diagnosis in the migraine group.

${ }^{*} p<0.025$.

${ }^{\star \star} p \leq 0.001$.

focal epilepsies $(p<0.025)$ (Table 5). Migraineurs with aura were clearly more sensitive to MFs than without aura $(p<0.025)$ (Table 6).

The significant results for the most discordant MFs between various countries, evaluated for triggers cited by more than 15 individuals to avoid bias, were given in Tables 7, 8 .

\section{DISCUSSION}

This large-sized and comprehensive transcultural survey draws attention to the relatively neglected topic of MFs, either provocative or inhibitory, which were comparatively investigated in 369 PWE and 239 PWM, in four culturally different countries using the same standardized method. Most subjects reported 
TABLE 7 | Discordant rates of attack modulatory factors in patients with epilepsy among the different countries.

\begin{tabular}{|c|c|c|c|c|}
\hline & $\begin{array}{c}\text { Brazil } \\
N=82\end{array}$ & $\begin{array}{l}\text { Turkey } \\
N=96\end{array}$ & $\begin{array}{c}\text { Guatemala } \\
N=42\end{array}$ & $\begin{array}{c}\text { Lithuania } \\
N=149\end{array}$ \\
\hline Certain memories* & $\underline{50.0}$ & 3.1 & 19 & 12.8 \\
\hline Certain thoughts* & 53.7 & 5.2 & 14.3 & 13.4 \\
\hline Déjà vu* & $\underline{24.4}$ & 0 & 9.5 & $\underline{22.1}$ \\
\hline Positive feelings - inhibition* & 9.8 & 0 & 0.8 & $\underline{18.8}$ \\
\hline Negative feelings ${ }^{\star}$ & $\underline{68.3}$ & 53.1 & $\underline{71.4}$ & 41.6 \\
\hline Thinking/concentration * & 34.1 & 6.3 & 19 & 9.4 \\
\hline Inhibition* & 3.7 & 0 & $\underline{16.7}$ & 11.4 \\
\hline Mental calculations* & $\underline{19.5}$ & 1 & 7.1 & 4 \\
\hline Alcohol* $^{*}$ & 19.5 & 12.5 & 9.5 & $\underline{27.5}$ \\
\hline (Not applicable) & 24.4 & 66.7 & 0 & $(2.7)$ \\
\hline Coffee $^{\star \star}$ & 6.3 & 1 & $0^{\star}$ & $\underline{11.8}$ \\
\hline (Not applicable) & 3.7 & 4.2 & 0 & (4) \\
\hline Sudden unexpected loud noise* & $\underline{23.2}$ & 0 & 14.3 & $\underline{18.1}$ \\
\hline Listening to talks, audit. overexposure * & $\underline{22}$ & 0 & 0 & 6.7 \\
\hline Pain* & $\underline{26.8}$ & 0 & $\underline{26.2}$ & 15.4 \\
\hline Emotional speaking ${ }^{\star \star}$ & 6.1 & 3.1 & $\underline{16.7}$ & $\underline{15.3}$ \\
\hline Reading silently* & 7.3 & 0 & 4.8 & 15.3 \\
\hline Inhibition* & 0 & 0 & 0 & 11.9 \\
\hline Sports* & $\underline{23.2}$ & 1 & 14.3 & 11.4 \\
\hline Excess sleep* & $\underline{19.5}$ & 0 & 0 & 10.1 \\
\hline Seizures upon awakening ${ }^{\star \star}$ & 17.1 & $\underline{27.1}$ & $\underline{31}$ & 9.4 \\
\hline Seizures while asleep* & $\underline{22}$ & 3.1 & 2.4 & 13.4 \\
\hline Physical stress* & $\underline{40.2}$ & $\underline{43.8}$ & 9.5 & 20.1 \\
\hline Fever $^{\star \star}$ & $\underline{22}$ & 7.3 & 19 & 11.4 \\
\hline
\end{tabular}

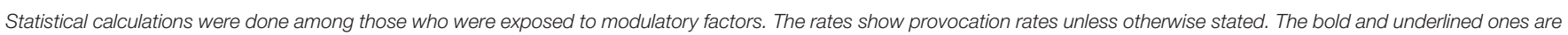

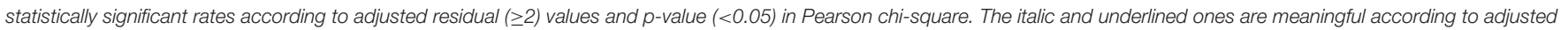
residuals and $p$-values $(<0.05)$ in chi-square, but not the rates in which the number of individuals are $<15$.

${ }^{*} p \leq 0.001$.

${ }^{\star *} p<0.05$.

PFs (91.6\% of PWM and $82.7 \%$ of PWE) at least once in their lifetime. These numbers agree with the literature, varying from 60 to $100 \%$ for migraine patients $(10-14)$ and 47 to $97 \%$ in PWE (15-21). The occurrence of MFs is well-known in both epilepsy and migraine but has not been comparatively assessed before. Migraine and epilepsy are chronic disorders with episodic attacks, and long recognized associations between them include some clinical features, including external and internal triggers, as well as some gene mutations $(22,23)$.

There is a well-known gender predominance in migraine worldwide, with women representing more than $80 \%$ of the patient population, similar to our study. In fact, migraine occurs approximately three times more often in women than in men (18, $24,25)$. Sex hormonal changes have major impacts particularly on migraine during lifetime; but the underlying mechanisms are not illuminated yet $(4,26)$. Diversely, Christensen et al. evaluated gender differences in epilepsy, finding no overall difference, but in subsequent analyses, they found that genetic generalized epilepsies were more frequent in women than in men (27). Similar to our findings, Ferlisi and Shorvon did not observe differences in frequency or type of seizure precipitants with regard to gender (15).
Several authors reported stress as the most prevalent PF reported by PWE (16-21), while in our study, it was the most prevalent PF reported in migraine. The first ranking PF among PWE was lack of sleep, similar to da Silva Sousa et al. (20). Sleep deprivation was also cited by other authors $(18,19)$. Certain thoughts and certain memories were not commonly assessed in other publications. da Silva Sousa et al. reported specific thoughts/concentration being recognized by $23 \%$ of the patients (20). In our study, certain thoughts and memories were reported in 20.3 and $19.2 \%$, respectively, of PWE.

Emotional stress was the most important PF reported by migraine patients $(81.6 \%)$, like other studies, where stress was reported by $48-84 \%$ probably related to the somatic effect of hyperexcitability on the autonomic nervous system (9-12, 28, 29).

Our PWM also mentioned negative feelings as a PF in $75.7 \%$. Hauge et al. found it in up to $58 \%$ (11). Also, lack of sleep was similarly reported high among $77.8 \%$ of migraineurs in our study; it was also identified in other surveys by $50-64 \%$ (28). Besides, the influence of dietary factors or fasting was cited by $44-58 \%$ (28). Within our population of PWM, 71.1\% reported hunger and $59 \%$ fasting as a triggering factor. It was remarkable that 
TABLE 8 | Discordant rates of attack modulatory factors in patients with migraine among the three different countries.

\begin{tabular}{|c|c|c|c|}
\hline & $\begin{array}{c}\text { Brazil } \\
(N=44) \%\end{array}$ & $\begin{array}{c}\text { Turkey } \\
(N=94) \%\end{array}$ & $\begin{array}{c}\text { Lithuania } \\
(N=101) \%\end{array}$ \\
\hline Certain memories* & 20.5 & 5.3 & $\underline{19.8}$ \\
\hline Certain thoughts* & $\underline{40.9}$ & 7.4 & $\underline{44.4}$ \\
\hline Positive feelings * inhibition & 13.6 & 3.2 & $\underline{29.7}$ \\
\hline Negative feelings ${ }^{\star}$ & $\underline{90.9}$ & 60.6 & 83.2 \\
\hline Thinking/concentration* & $\underline{61.4}$ & 33 & $\underline{62.4}$ \\
\hline Decision-making ${ }^{\star \star} \neq$ & $\underline{47.7}$ & 27.7 & 25.7 \\
\hline Alcohol* $^{*}$ & 18.2 & $\underline{14.9}$ & $\underline{44.6}$ \\
\hline (Not applicable) & 27.3 & 70.2 & (2) \\
\hline Coffee* $^{*}$ & 14.6 & 11.9 & $\underline{21.8}$ \\
\hline Inhibition & $\underline{26.8}$ & 7.1 & 19.8 \\
\hline (Not applicable) & 6.8 & 10.6 & (0) \\
\hline Smoking $^{\star}$ & 0 & 21.4 & $\underline{33.3}$ \\
\hline (Not applicable) & 61.4 & 55.3 & (2) \\
\hline Another subst* & 6.8 & 0 & $\underline{15.3}$ \\
\hline (Not applicable) & 93.2 & 98.9 & (3) \\
\hline Chewing $^{\star}$ & 4.5 & 0 & $\underline{22.8}$ \\
\hline Fasting $^{\star *}$ & $\underline{81.8}$ & 54.3 & 53.5 \\
\hline Pleasant taste* & 2.3 & $\underline{22.3}$ & 3 \\
\hline Special taste or aroma* & 81.8 & 27.7 & 54.5 \\
\hline Flashes $^{\star \star}$ & 36.4 & 28.7 & $\underline{51.5}$ \\
\hline Closing the eyes (Inhibition) ${ }^{\star \star}$ & 27.3 & 20.2 & $\underline{43.6}$ \\
\hline $\mathrm{TV}^{*}$ & 38.6 & 10.6 & $\underline{43.6}$ \\
\hline Any song* & $\underline{40.9}$ & 0 & 0 \\
\hline Specific pitch* & $\underline{40.9}$ & 5.3 & $\underline{44.6}$ \\
\hline $\begin{array}{l}\text { Listening to talks, audit. } \\
\text { overexposure }^{\star}\end{array}$ & $\underline{65.9}$ & 18.1 & 43.6 \\
\hline Specific voices* & 11.4 & 3.2 & $\underline{44.6}$ \\
\hline Phone ringing, answering, etc. ${ }^{*}$ & 15.9 & 7.4 & 34.7 \\
\hline Certain rhythms* & 34.1 & 2.1 & 23.8 \\
\hline Pain* & 10.9 & 0.4 & $\underline{24.3}$ \\
\hline Certain own movements* & $\underline{45.5}$ & 1.1 & 31.7 \\
\hline Videogames, playstation* & 7 & $\underline{21.6}$ & $\underline{26.7}$ \\
\hline (Not applicable) & 40.9 & 60.6 & (1) \\
\hline Work on computer* & 35.8 & 37.3 & $\underline{61.4}$ \\
\hline (Not applicable) & 11.4 & 20.2 & (0) \\
\hline Public speaking* & 6.8 & 5.3 & $\underline{46.5}$ \\
\hline Writing $^{\star}$ & $\underline{13.6}$ & 2.1 & $\underline{14.9}$ \\
\hline Bathing, hot water inhibition* & 9.1 & 6.4 & $\underline{33.7}$ \\
\hline Sports* & 15.9 & 13.8 & $\underline{25.7}$ \\
\hline Inhibition* & 9.1 & 0 & $\underline{14.9}$ \\
\hline Singing ${ }^{\star}$ & 2.3 & 0 & $\underline{15.1}$ \\
\hline Dancing* & 6.8 & 0 & $\underline{15.1}$ \\
\hline Sexual activity inhibition* & 0 & 0 & $\underline{23.2}$ \\
\hline Orgasm inhibition* & 4.5 & 2.1 & $\underline{23.2}$ \\
\hline Excess sleep ${ }^{\star \star}$ & 45.5 & 45.7 & $\underline{67.3}$ \\
\hline Seizures upon awakening* & $\underline{72.7}$ & 3.2 & $\underline{61.4}$ \\
\hline Seizures while asleep* & 25 & 0 & $\underline{57.4}$ \\
\hline Physical stress* & $\underline{81.8}$ & 34 & 65.3 \\
\hline Emotional stress ${ }^{\star \star}$ & 93.2 & 83 & 75.2 \\
\hline
\end{tabular}

(Continued)
TABLE 8 | Continued

\begin{tabular}{lccc}
\hline & $\begin{array}{c}\text { Brazil } \\
(\mathbf{N}=\mathbf{4 4 )})\end{array}$ & $\begin{array}{c}\text { Turkey } \\
(\mathbf{N}=\mathbf{9 4}) \%\end{array}$ & $\begin{array}{c}\text { Lithuania } \\
(\mathbf{N}=\mathbf{1 0 1}) \%\end{array}$ \\
\hline Fever $^{\star}$ & 22.7 & 3.2 & $\underline{\mathbf{5 8 . 4}}$ \\
Menstruation $^{\star}$ & $\underline{\mathbf{8 6 . 1}}$ & 58.2 & $\underline{\mathbf{6 8}}$ \\
(Not applicable) $^{\text {No.2 }}$ & 18.2 & 36.2 & $(1)$ \\
\hline
\end{tabular}

Statistical calculations were done among those who were exposed to modulatory factors. The rates show provocation rates unless otherwise stated. The bold and underlined ones are statistically significant rates according to adjusted residual $(\geq 2)$ values and $p$-value $(<0.05)$ in Pearson chi-square. The italic and underlined ones are meaningful according to adjusted residuals and p-values $(<0.05)$ in chi-square, but not the rates in which the number of individuals are $<15$.

Audit, auditory.

$\neq$ Decision-making between different possible things to do.

${ }^{*} p \leq 0.001$.

${ }^{* *} p<0.05$

the dietary control of individual-specific food triggers reduced the number of monthly attacks of migraine in a double-blind, crossover, randomized controlled trial, showing evidence for the importance of searching and controlling the relevant MF, as an additional management option (30).

As expected, modulation of attacks by light sources was identified in higher rates among PWM (52.7\% of PWM vs. $18.7 \%$ of PWE), related possibly to photophobia in relation to migraine. Also, menstruation was a very important $\mathrm{PF}$ among PWM (68.4\%) and of less importance among PWE $(18.9 \%)$, as consistent with literature $(26,31)$. An interesting and unexpected significant difference between migraine and epilepsy triggers was "fever" (13.5\% of PWE vs. $30.1 \%$ of PWM reported attacks triggered by fever). Fever is a well-known trigger in epilepsy (32), and also headaches related to fever were reported in the Headache Classification-ICHD (2018), but there is not much known about fever triggering migraine episodes (8).

In our subgroup comparisons of PWE, lights, lack of sleep, and seizure upon awakening were reported more frequently among generalized epilepsies, whereas emotional, movement, and auditory-related triggers were frequent among focal epilepsies, as reported by others in different studies $(5,33)$.

Our migraineurs with aura reported a more sensitive picture to triggers. More frequently reported PFs among migraineurs with aura were as follows: visual triggers like lights and striped patterns; physical stress; certain memories; some auditory triggers; and games like chess/cards. Sexual activity and orgasm reported to be both inhibitory and provocatory on attacks more frequently in MWA than MWOA. Similar to ours, Kelman reported also migraineurs with aura were more sensitive to some triggers including lights (12). But there are some other studies showing different results. Rasmussen and Olesen found the frequency of various PFs was higher in migraineurs without aura (34), and Russel et al. only found lights more frequent among migraineurs with aura (35).

Perhaps the most remarkable finding in this direct comparison of epilepsy and migraine triggers is the identical appearance of the three leading provocative factors: negative feelings, emotional 
stress, and lack of sleep. It can be discussed to what extent these are real factors or, rather, subjective attributions. That negative feelings and their counterpart, positive feelings (which have a similar place among inhibitors), that appear in these roles may appear trivial; they may very well be prodromal symptoms rather than modulating factors. But they may also reflect still unrecognized systemic dynamics as factors of symptom generation that are common to both disorders.

To discover factors specific for the different conditions, it may be that we have primarily to look into less frequent observations where migraine and epilepsy differ. Thus, when we disregard the commonly reported influences of positive and negative feelings and the apparently specific effect of coffee in migraine, it appears that IFs in epilepsy primarily have something to do with increased concentration and arousal, but those in migraine have something to do with relaxation and reduced sensory input.

Indeed, we were expecting to show that reported PFs would be less frequent and IFs would be more frequent among elderly PWE; however, we could not show this. Mnemonic triggers (26.4\%), negative feelings (61.2\%), auditory overexposure (11.2\%), and sexual activity (7.2\%) were more frequent PFs among the elderly PWE, whereas lights (23.7\%), TV(15.3\%), videogames (16.4\%), and computer (18.7\%) were reported PFs among the younger group. This photic sensitivity is a well-known phenomenon among the younger PWE, especially in the genetic generalized epilepsy (GGE), and our survey confirms this (36). Interestingly, dancing (3.2\%) and sexual activity (2.8\%) were IFs reported more frequently among the younger PWE group, although the rates were not high. It is obvious that systematic prospective studies are needed to investigate the frequency and characteristics of triggers in epilepsy at different age ranges and during the course of the disease.

Younger migraineurs were more sensitive to MFs. On the other hand, reported rates of IFs were not higher among elderly PWM, which we were expecting to be. For instance, emotional stress has not been reported at divergent rates between elderly and younger PWE groups, and what even more surprising was that positive feelings were reported more frequently as an IF among the younger PWM (23\%). The other frequent IFs among younger PWM were as follows: sexual activity (16.2\%), pleasant taste $(9.8 \%)$, drawing $(9.8 \%)$, singing $(7.4 \%)$, certain rhythms (5.7\%), writing $(6.6 \%)$, and pain (3.3\%). On the other hand, frequently reported PFs among younger PWE were writing (69.6\%), excess sleep (63.9\%), pain $(44.3 \%)$, public speaking (32.8\%), seizures while asleep (37.7\%), fever (42.6\%), specific voices $(29.5 \%)$, and chewing $(17.2 \%)$. The only more frequently reported trigger among the elderly group was unpleasant aroma (60\%). Most probably, these divergent rates among the younger and elderly groups may be associated with development of trigger avoidance or desensitization strategies by time. There is a lack of studies investigating the impact of age and disease duration on migraine triggers (37).

The incidence of MFs for migraine and seizures across different countries demonstrated the clinical consistency of these conditions. However, the top first PF and IF were slightly different across the sites, suggesting that regional cultural characteristics and beliefs may have influenced the answers (38,
39), as previously found by Asadi-Pooya and Sperling when they compared seizure precipitants between a Middle Eastern country (Iran) and a Western country (USA) (40). Interestingly, a series of remarkable differences were found between the four participating countries (see Tables 7, 8). Certain memories (50\%) and thoughts (53.7\%), mental calculations (19.5\%), thinking/concentration (34.1\%), fever (22\%), pain (26.8\%), sports $(23.2 \%)$, excess sleep (19.5\%), and auditory factors (23\%) were reported strikingly higher as being provocative for seizures in Brazilian PWE than PWE in other countries for unknown reasons. In Lithuanian PWE, alcohol (26\%), coffee (11.4\%), emotional speaking (15.7\%), and reading silently (15.3\%) were conspicuous PFs, whereas in Turkish PWE, physical stress (40.2\%) and awakening (27.1\%) were the significantly noted features. Negative feelings was the most reported PF among Guatemalans (71.4\%) and followed by the Brazilian (68.3\%) PWE. The other interesting point was the higher reporting rates of positive feelings $(18.8 \%)$, reading silently $(11.9 \%)$, and thinking/concentration (11.4\%) as IFs among the Lithuanian PWE.

Furthermore, when the reported MFs of PWM among countries were compared, there were also interesting differences. The triggering stimuli, like certain memories (19.8\%), coffee (21.8\%), another substance (14.9\%), and chewing (22.8\%) were significantly common among Lithuanian PWM; decision making (47.7\%), fasting $(81.8 \%)$, special taste or aroma $(81.8 \%)$, any song $(40.9 \%)$, specific pitch (40.9\%), listening to talks $(65.9 \%)$, certain rhythms (34.1\%), certain own movements (45.5\%), awakening (72.7\%), and physical stress $(81.8 \%)$ were reported in discordant rates among Brazilian PWM. In Turkish PWM, pleasant tastes $(22.3 \%)$ were reported as provocatory in higher rates. It was also shown that the same factors can act in opposite directions; positive feelings (29.7\%), coffee (19.8\%), sports (14.9\%), sexual activity (22.8\%), and orgasm (22.8\%) were the outstanding IFs reported to be higher among Lithuanian PWM. All these discordant rates might be explained by the sociocultural differences, the way of perceiving questions in four different languages, as the terms may have slightly different connotations. Additionally for those PWE, discordances might also be explained by the fact of different prevalence of focal and generalized epilepsies among patients from different countries [e.g., focal epilepsy was present in different rates among Brazilian (92.7\%), Lithuanians (67.1\%) Turkish (36.5\%), and Guatemalans (64.3\%)]. We also want to emphasize that our findings underlined the fact that individualized approach is important to help the patients.

A very important question is whether perceptions of protective factors enable patients to perceive some voluntary control over their seizures or migraine attacks $(41,42)$. Lunardi et al. found that $50.7 \%$ of the patients identified at least one IF in a cohort of temporal lobe epilepsy (17). Moreover, 82 (22.8\%) PWE and $45(18.8 \%)$ PWM reported being able to prevent or arrest their episodes, and results were concordant with a previous study, where $23 \%$ of the patients revealed they were capable of avoiding the occurrence of their seizures (20). Pinikahana and Dono revealed that $69.8 \%$ from an epilepsy research database indicated that they had tried at least one technique to stop a seizure, with resting, acute medication use, and relaxation being the most 
common (18). Another study reported that $47 \%$ of the subjects could sometimes stop their seizures from happening, mostly by relaxation techniques (21). The most commonly reported behaviors of stopping the migraine attacks are rather non-specific like isolation from light, sound (75-95\%), lying down (65-89\%), and sleeping (60-89.3\%); and therefore, causality establishment is not easy. Thus, the exact rate of PWM who could stop their attacks could not be stated appropriately (42).

On the contrary, when the induction of seizures was evaluated, only 18 (5\%) of PWE and 38 (16.3\%) of those with migraine informed that they could provoke a seizure or migraine attack. Cull et al. reported that $8.9-9.7 \%$ of their cohort could induce seizures (43). Pinikahana and Dono revealed a larger number: about $35.1 \%$ of their population could trigger a seizure voluntarily (18). Self-provocation in migraine is a neglected issue, but it has been reported that attacks/auras could be triggered by pharmacological and non-pharmacological stimuli $(44,45)$. Hougaard et al. reported that only $11 \%$ of the attacks were triggered by exercise in the laboratory environment, but none by light stimulation in PWM, who already reported that their attacks were triggered by light and exercise in daily life (46). These studies reflect the complex nature of the initiation of migraine.

Our study has some limitations: First of all, to make the distinctions clearer, the highly prevalent situation of comorbidity between migraine and epilepsy was not systematically assessed in our population, and those cases with comorbidity were not included (47).The questionnaire used here was constructed as an instrument that could be applied for both investigated disease conditions, for the sake of this study. In addition, there is a lack of gold-standard tests to validate our questionnaire with. Although validation is recommended in all questionnaires in the health field to ensure that the questionnaire is psychometrically sound, it may be argued by some authors (48), and most of the questionnaires previously published on the subject of MFs have not been validated yet.

Theoretically, episode prediction currently rests on the identification of trigger factors and protective factors. This task may be misleading, as the terms "trigger factors" ("measureable endogenous or exogenous events/exposures associated with an increased probability of an attack over a relatively brief period of time, with examples such as menses") and "premonitory features" ("precede the attack by up to $48 \mathrm{~h}$ and may include cognitive and behavioral factors, such as feeling tired/weary, concentration difficulties") are often confused in the literature (39), compromising the reproducibility of these numbers across different studies.

Several items of our questionnaire could be criticized, though reflecting real-life usage, being not to clearly distinguish between these two possibilities. They were, however, kept in, as we otherwise could have lost important information on patients' subjective experiences. For instance, it is not clear if all patients from different countries with different diagnoses mean the same thing when they give an answer to a question, such as déjà vu or negative feelings. However, this is a limitation inherent to all questionnaires; they are all subject to patients' interpretation.

Furthermore, other limitations, characteristic of all psychosocial studies, are worthwhile to mention. As already said above, in studies like this based on self-reported or researcher-administrated surveys, the collected information may be influenced by beliefs, sociocultural levels, affective disorders, or symptoms and also recall bias. While questioning the triggers of the patients, it might be useful to correlate them with anxiety and depression scales, but as it was not our main purpose in this study, we did not accede to them. On the other hand, such as the predominance of women among the migraineurs, age of onset, and hence the disease duration, frequency of episodes was indeed different between groups since epilepsy and migraine have a little bit distinct nature. Also, in such a large series of cases, the non-matching age distribution and durations of diseases between countries can be considered among the limitations. Nonetheless, such studies are important for hypothesis generation, while being able to assess beliefs about triggers and premonitory features and multiple other factors. Besides, to identify MFs from a checklist may limit only to candidate factors with available data, although our questionnaire was intended to be comprehensive with more than 60 items. Also, this modality of study may not distinguish between causality and reverse causality (39), while it is not time-consuming and it may be easily applied in different and large populations. At last, this study was conducted mostly in tertiary referral centers, which may have biased the population toward more severe epilepsy or migraine conditions.

The reliable recognition of opportunities to reduce episode frequency such as trigger identification and avoidance, preemptive therapy (either pharmacologic or behavioral approaches), and enhancing timely protective factors may, in some cases, be the mainstay of the treatment, or being important as an adjunctive measure alongside the regular pharmacological treatment, since the unpredictability of migraine or seizure episodes is one of the most incapacitating features of these conditions $(39,49)$. Further studies assessing the roles of MFs in specific epilepsy syndromes as well as other migraine subtypes with more homogeneous patient populations will provide more useful information.

\section{CONCLUSION}

The great majority of patients identified PFs for both their epileptic seizures and migraine attacks, while one in three patients recognized that their episodes could be inhibited by specific measures. PWM report higher sensitivity to triggers than the PWE, when investigated with the same standardized questionnaire, for the first time. Interestingly, the same stimulus can both precipitate and abort a seizure in different individuals or even in the same individual. This phenomenon does not have any explanation so far; it may depend on the state of cortical network activation at the moment the input is given in a susceptible person. A better understanding of these MFs may provide insights into disease pathophysiology, as also the knowledge of seizure precipitants may empower patients in increasing their self-awareness, by promoting behavioral modification with avoidance of specific high-risk situations and, potentially, a reduction in seizure/migraine episodes. We also showed that these MFs are mostly concordant across 
the countries only with some minor transcultural changes, which further indicated that they are reliable means for future investigations. Proper recognition of seizure/migraine trigger factors and other MFs could be helpful in routine daily practice, leading to new and personalized treatment strategies, besides helping to create homogenous groups for advanced genetic and neuroimaging researches.

\section{DATA AVAILABILITY STATEMENT}

The original contributions presented in the study are included in the article/Supplementary Material, further inquiries can be directed to the corresponding author/s.

\section{ETHICS STATEMENT}

The studies involving human participants were reviewed and approved by Universidade Federal de Santa Catarina Ethics Committee, CEPSH/UFSC N. 1.226.636 (14/09/2015); Istanbul Medical Faculty Ethics Committee, n ${ }^{\circ}$ 26/02/16/26204; Investigation Committee of the Epilepsy and Functional Neurosurgery Center Humana CENFHU-06-2015; Vilnius Regional Biomedical Research Ethics Committee $\mathrm{n}^{\circ}$ 158200-15797-309, 2015-09-07. Written informed consent to participate in this study was provided by the participants' legal guardian/next of kin.

\section{AUTHOR CONTRIBUTIONS}

$\mathrm{BB}, \mathrm{EU}, \mathrm{KL}, \mathrm{RM}, \mathrm{RS}$, and PW contributed to the conception and design of the study. EU, KL JS, HS, RM, RS, LV, AF, JA, MS, LQ, and EZ contributed to data collection. KL and EU organized the database and performed the statistical analysis. $\mathrm{KL}, \mathrm{EU}, \mathrm{BB}$, and PW wrote the first draft of the manuscript. All authors contributed to manuscript draft, read, and approved the submitted version.

\section{FUNDING}

Global Migraine and Pain Society, Istanbul, Turkey supported this study. The Prof. Dr. Peter and Jytte Wolf Foundation

\section{REFERENCES}

1. Yeh WZ, Blizzard L, Taylor BV. What is the actual prevalence of migraine? Brain Behav. (2018) 8:e00950. doi: 10.1002/brb3.950

2. Baykan B, Ertas M, Karli N, Uluduz D, Uygunoglu U, Ekizoglu $\mathrm{E}$, et al. Migraine incidence in 5 years: a population-based prospective longitudinal study in Turkey. J Headache Pain. (2015) 16:103. doi: 10.1186/s10194-015-0589-2

3. Bell GS, Neligan A, Sander JW. An unknown quantity-the worldwide prevalence of epilepsy. Epilepsia. (2014) 55:958-62. doi: 10.1111/epi.12605

4. Onal AE, Tumerdem Y, Ozturk MK, Gurses C, Baykan B, Gokyigit A, et al. Epilepsy prevalence in a rural area in Istanbul. Seizure. (2002) 11:397401. doi: 10.1053/seiz.2001.0665

5. Mameniškiene R, Wolf P. Precipitation and inhibition of seizures in focal epilepsies. Expert Rev Neurother. (2018) 18:275-87. doi: 10.1080/14737175.2018.1455502 for Epilepsy, Bielefeld, Germany, supported the EpExMo cooperation and contributed to the publication fees. KL was supported by PRONEM (Programa de Apoio a Nucleos Emergentes - KETODIET-SC Project - Process No 2020TR736) from FAPESC/CNPq, Santa Catarina, Brazil. The content is solely the responsibility of the authors.

\section{ACKNOWLEDGMENTS}

This work is part of the EpExMo cooperation, an open international research initiative for the study of epilepsies with external modulation of ictogenesis (EpExMo). The participating centers of this study are the following: Danish Epilepsy Center, Dianalund, Denmark (SB and PW); Instituto de Neurología, Hospital de Clínicas, Universidad de la República, Montevideo, Uruguay (PB, AB, and AS); Epilepsy Center of Santa Catarina (CEPESC) and Federal University of Santa Catarina (UFSC), Florianopolis, Brazil (ML, LC, RW, and KL); Unidade de Pesquisa e Tratamento das Epilepsias and Department of Neurology and Neurosurgery, Federal University of São Paulo, São Paulo, Brazil (YE and MSBG); Epilepsy Center, Vilnius University Hospital Santariškiu Klinikos, Vilnius, Lithuania (RM); Children's Hospital, an affiliate of Vilnius University Hospital Santariškiu Klinikos, Vilnius, Lithuania (RS); and the Department of Neurology and Clinical Neurophysiology, Istanbul Medical Faculty, Istanbul University, Istanbul, Turkey (BB). KL holds a PQ2 Research Fellowship from Conselho Nacional de Desenvolvimento Científico e Tecnológico (CNPq; process no. 304936/2017-0). PW holds a Special Visiting Researcher Fellowship in Brazil-Programa Ciência sem Fronteiras from Coordenação de Aperfeiçoamento de Pessoal de Nível Superior (CAPES; project MEC/MCTI/CAPES/CNPq/FAPs no. 88881.030478/2013-01). The authors also thank to the Global Migraine and Pain Society and the Prof. Dr. Peter and Jytte Wolf Foundation for Epilepsy.

\section{SUPPLEMENTARY MATERIAL}

The Supplementary Material for this article can be found online at: https://www.frontiersin.org/articles/10.3389/fneur. 2021.672860/full\#supplementary-material
6. Mackay M, Mahlaba H, Gavillet E, Whittaker RG. Seizure self-prediction: myth or missed opportunity? Seizure. (2017) 51:180-5. doi: 10.1016/j.seizure.2017.08.011

7. Marmura MJ. Triggers, protectors, and predictors in episodic migraine. Curr Pain Headache Rep. (2018) 22:81. doi: 10.1007/s11916-018-0734-0

8. Headache Classification Committee of the International Headache Society (IHS) The International Classification of Headache Disorders, 3rd edition. Cephalalgia. (2018) 38:1-211. doi: 10.1177/0333102417 738202

9. Scheffer IE, Berkovic S, Capovilla G, Connolly MB, French J, Guilhoto L, et al. ILAE classification of the epilepsies: position paper of the ILAE Commission for Classification and Terminology. Epilepsia. (2017) 58:51221. doi: 10.1111/epi.13709

10. Pavlovic JM, Buse DC, Sollars CM, Haut S, Lipton RB. Trigger factors and premonitory features of migraine attacks: summary of studies. Headache. (2014) 54:1670-9. doi: 10.1111/head.12468 
11. Hauge AW, Kirchmann M, Olesen J. Characterization of consistent triggers of migraine with aura. Cephalalgia. (2011) 31:416-38. doi: 10.1177/0333102410382795

12. Kelman L. The triggers or precipitants of the acute migraine attack. Cephalalgia. (2007) 27:394-402. doi: 10.1111/j.1468-2982.2007.01303.x

13. Andress-Rothrock D, King W, Rothrock J. An analysis of migraine triggers in a clinic-based population. Headache. (2010) 50:1366-70. doi: 10.1111/j.1526-4610.2010.01753.x

14. Baldacci F, Vedovello M, Ulivi M, Vergallo A, Poletti M, Borelli P, et al. How aware are migraineurs of their triggers? Headache. (2013) 53:8347. doi: $10.1111 /$ head. 12083

15. Ferlisi M, Shorvon S. Seizure precipitants (triggering factors) in patients with epilepsy. Epilepsy Behav. (2014) 33:101-5. doi: 10.1016/j.yebeh.2014.02.019

16. Wassenaar M, Kasteleijn-Nolst Trenité DG, de Haan GJ, Carpay JA, Leijten FS. Seizure precipitants in a community-based epilepsy cohort. J Neurol. (2014) 261:717-24. doi: 10.1007/s00415-014-7252-8

17. Lunardi Mdos S, Sukys-Claudino L, Guarnieri R, Walz R, Lin K. Seizure precipitants and inhibiting factors in mesial temporal lobe epilepsy. J Neurol Sci. (2011) 308:21-4. doi: 10.1016/j.jns.2011.06.041

18. Pinikahana J, Dono J. Age and gender differences in initial symptoms and precipitant factors of epileptic seizures: an Australian study. Epilepsy Behav. (2009) 16:231-39. doi: 10.1016/j.yebeh.2009.06.018

19. Nakken KO, Solaas MH, Kjeldsen MJ, Friis ML, Pellock JM, Corey LA. Which seizure-precipitating factors do patients with epilepsy most frequently report? Epilepsy Behav. (2005) 6:85-9. doi: 10.1016/j.yebeh.2004.11.003

20. da Silva Sousa P, Lin K, Garzon E, Sakamoto AC, Yacubian EM. Selfperception of factors that precipitate or inhibit seizures in juvenile myoclonic epilepsy. Seizure. (2005) 14:340-6. doi: 10.1016/j.seizure.2005.04.007

21. Spector S, Cull C, Goldstein LH. Seizure precipitants and perceived self-control of seizures in adults with poorly-controlled epilepsy. Epilepsy Res. (2000) 38:207-16. doi: 10.1016/S0920-1211(99) 00093-5

22. Dichgans M, Freilinger T, Eckstein G, Babini E, Lorenz-Depiereux B, Biskup S., et al. Mutation in the neuronal voltage-gated sodium channel SCN1A in familial hemiplegic migraine. Lancet. (2005) 366:3717. doi: 10.1016/S0140-6736(05)66786-4

23. Reiffurth C, Alam M, Zahedi-Khorasani M, Major S, Dreier JP. $\mathrm{Na}^{+} / \mathrm{K}^{+}$-ATPase $\alpha$ isoform deficiency results in distinct spreading depolarization phenotypes. J Cereb Blood Flow Metab. (2020) 40:622-638. doi: 10.1177/0271678X19833757

24. Peterlin BL, Gupta S, Ward TN, Macgregor A. Sex matters: evaluating sex and gender in migraine and headache research. Headache. (2011) 51:83942. doi: 10.1111/j.1526-4610.2011.01900.x

25. Dodick DW. Migraine. Lancet. (2018) 391:131530. doi: 10.1016/S0140-6736(18)30478-1

26. Karli N, Baykan B, Ertaş M, Zarifoglu M, Siva A, Saip S, et al. Impact of sex hormonal changes on tension-type headache and migraine: a crosssectional population-based survey in 2,600 women. J Headache Pain. (2012) 13:557-65. doi: 10.1007/s10194-012-0475-0

27. Christensen J, Kjeldsen MJ, Andersen H, Friis ML, Sidenius P. Gender differences in epilepsy. Epilepsia. (2005) 46:95660. doi: 10.1111/j.1528-1167.2005.51204.x

28. Mollaoglu M. Trigger factors in migraine patients. J Health Psychol. (2013) 18:984-94. doi: 10.1177/1359105312446773

29. Haque B, Rahman KM, Hoque A, Hasan AT, Chowdhury RN, Khan SU., et al. Precipitating and relieving factors of migraine versus tension type headache. BMC Neurol. (2012) 12:82. doi: 10.1186/1471-2377-12-82

30. Alpay K, Ertas M, Orhan EK, Ustay DK, Lieners C, Baykan B. Diet restriction in migraine, based on IgG against foods: a clinical double-blind, randomised, cross-over trial. Cephalalgia. (2010) 30:82937. doi: $10.1177 / 0333102410361404$

31. Reddy DS. Catamenial epilepsy: discovery of an extrasynaptic molecular mechanism for targeted therapy. Front Cell Neurosci. (2016) 10:101. doi: 10.3389/fncel.2016.00101

32. Whelan H, Harmelink M, Chou E, Sallowm D, Khan N, Patil R, et al. Complex febrile seizures-A systematic review. Dis Mon. (2017) 63:523. doi: 10.1016/j.disamonth.2016.12.001
33. Italiano D, Ferlazzo E, Gasparini S, Spina E, Mondello S, Labate A, et al. Generalized versus partial reflex seizures: a review. Seizure. (2014) 23:51220. doi: 10.1016/j.seizure.2014.03.014

34. Rasmussen BK, Olesen J. Migraine with aura and migraine without aura: an epidemiological study. Cephalalgia. (1992) 12:221-8. doi: 10.1046/j.1468-2982.1992.1204221.x

35. Russell MB, Rasmussen BK, Fenger K, Olesen J. Migraine without aura and migraine with aura are distinct clinical entities: a study of four hundred and eighty-four male and female migraineurs from the general population. Cephalalgia. (1996) 16:239-45. doi: 10.1046/j.1468-2982.1996.1604239.x

36. Martins da Silva A, Leal B. Photosensitivity and epilepsy: current concepts and perspectives-A narrative review. Seizure. (2017) 50:20918. doi: 10.1016/j.seizure.2017.04.001

37. Peris F, Donoghue S, Torres F, Mian A, Wöber C. Towards improved migraine management: determining potential trigger factors in individual patients. Cephalalgia. (2017) 37:452-63. doi: 10.1177/0333102416649761

38. Moritz JLW, Mameniškiene R, Rimšiene J, Budriuniene A, de Almeida Calado G, et al. Control perceptions in epilepsy: a transcultural casecontrol study with focus on auras. Epilepsy Behav. (2018) 88:1308. doi: 10.1016/j.yebeh.2018.09.005

39. Lipton RB, Pavlovic JM, Haut SR, Grosberg BM, Buse DC. Methodological issues in studying trigger factors and premonitory features of migraine. Headache. (2014) 54:1661-9. doi: 10.1111/head.12464

40. Asadi-Pooya AA, Sperling MR. Do foods precipitate seizures? A cross-cultural comparison. Epilepsy Behav. (2007) 11:4503. doi: 10.1016/j.yebeh.2007.07.004

41. Wolf P. From precipitation to inhibition of seizures: rationale of a therapeutic paradigm. Epilepsia. (2005) 46):15-6. doi: 10.1111/j.0013-9580.2005.461005.x

42. Bag B, Karabulut N. Pain-relieving factors in migraine and tension-type headache. Int J Clin Pract. (2005) 59:7603. doi: 10.1111/j.1368-5031.2005.00535.x

43. Cull CA, Fowler M, Brown SW. Perceived self-control of seizures in young people with epilepsy. Seizure. (1996) 5:1318. doi: 10.1016/S1059-1311(96)80107-5

44. Martin PR, Reece J, Forsyth M. Noise as a trigger for headaches: relationship between exposure and sensitivity. Headache. (2006) 46:96272. doi: 10.1111/j.1526-4610.2006.00468.x

45. Lindblad M, Hougaard A, Amin FM, Ashina M. Can migraine aura be provoked experimentally? A systematic review of potential methods for the provocation of migraine aura. Cephalalgia. (2017) 37:74-88. doi: 10.1177/0333102416636097

46. Hougaard A, Amin FM, Hauge AW, Ashina M, Olesen J. Provocation of migraine with aura using natural trigger factors. Neurology. (2013) 80:42831. doi: 10.1212/WNL.0b013e31827fof10

47. Nunes JC, Zakon DB, Claudino LS, Guarnieri R, Nunes FC, Queiroz LP., et al. Headache among mesial temporal lobe epilepsy patients: a case-control study. J Neurol Sci. (2011) 306:20-3. doi: 10.1016/j.jns.2011.04.006

48. Hawkins M, Elsworth GR, Osborne RH. Questionnaire validation practice: a protocol for a systematic descriptive literature review of health literacy assessments. BMJ Open. (2019) 9:e030753. doi: 10.1136/bmjopen-2019-030753

49. Lin K, Guaranha M, Wolf P. Reflex epileptic mechanisms in ictogenesis and therapeutic consequences. Expert Rev Neurother. (2016) 16:57385. doi: $10.1586 / 14737175.2016 .1169174$

Conflict of Interest: The authors declare that the research was conducted in the absence of any commercial or financial relationships that could be construed as a potential conflict of interest.

Copyright (C) 2021 Ur Özçelik, Lin, Mameniškienè, Sauter Dalbem, Siqueira, Samaitiene, Vega Zeissig, Fonseca, Mazini Alves, dos Santos Lunardi, de Queiroz Zubavičiuté, Wolf and Baykan. This is an open-access article distributed under the terms of the Creative Commons Attribution License (CC BY). The use, distribution or reproduction in other forums is permitted, provided the original author $(s)$ and the copyright owner(s) are credited and that the original publication in this journal is cited, in accordance with accepted academic practice. No use, distribution or reproduction is permitted which does not comply with these terms. 